\title{
Cooperative Combination Recognition of Computer Science and Information Science Based on Open Unrelated Knowledge Co-Occurrence Network
}

\author{
Yunxing Chen, Yuhua Wei \\ Guangzhou Huali Science and Technology Vocation College, Guangzhou, Guangdong, China \\ 188886061@qq.com
}

Keywords: Unrelated knowledge, Collinear network, Computer science, Informatics, Cooperative portfolio recognition

\begin{abstract}
Based on the open and unrelated knowledge co-occurrence network method, taking information science and computer science as examples, it identifies potential interdisciplinary collaborative research topics in the two disciplines.Based on the interdisciplinary keyword cooccurrence network, a two-dimensional vector space model of keywords in the network is constructed. According to certain principles, the middle words define the substitution method of the keyword vector model to identify the potential interdisciplinary collaborative research topics of keywords. Using the open and unrelated knowledge discovery method proposed in this paper, the potential interdisciplinary collaborative research topics of information science and computer science were discovered in the empirical research. This method can provide reference for interdisciplinary cooperation.
\end{abstract}

\section{Introduction}

In recent years, knowledge research has been continuously refined, and many scientific research projects or major problems have been solved in interdisciplinary disciplines ${ }^{[1]}$.In the process of interdisciplinary cooperation, it is the prerequisite to carry out interdisciplinary cooperation that the theme of interdisciplinary cooperation research that suits you is the key to the success of interdisciplinary cooperation ${ }^{[2]}$. Based on this, the author used information science and computer science as examples in the previous research, and applied closed non-related knowledge discovery methods to identify potential interdisciplinary collaborative research topics in the two disciplines.

At present, there are two main types of research on interdisciplinary cooperation. One type discusses the development mechanism and influencing factors of interdisciplinary cooperation from a perspective ${ }^{[3]}$.Combined the cases and found that the internal work in the interdisciplinary cooperation depends on the disciplines of the members ${ }^{[4]}$.Designed a measurement framework for the evolution characteristics of interdisciplinary cooperation, generic methods have measure the interdisciplinarity and comprehensive compactness indicators of the research field.There are more and more researches on the interdisciplinary cooperation theories and models, and the measurement of the degree of cooperation, but there is almost no research on the identification of potential themes of interdisciplinary cooperation ${ }^{[5]}$.Therefore, this article uses open non-related knowledge discovery methods to identify potential interdisciplinary collaborative research topics, and provides scholars with suggestions on the direction of cooperation in interdisciplinary cooperation.

\section{Research Methods Information Science and Computer Science Potential Interdisciplinary Collaborative Research Topics}

\subsection{Data Collection and Preprocessing}

This article uses the three journals with the highest composite impact factor in the field of information science. The sample data marks the source of the sample data 4605 keywords. Only the keywords that appear in the field of information science are suffixed, and the keywords that appear in the field of computer science do not add a suffix. Therefore, the keywords shared by information 
science and computer science connect the keyword co-occurrence networks of the two disciplines to form an interdisciplinary keyword co-occurrence network.

\subsection{Two-Dimensional Vector Space Model of Keywords}

The keyword co-occurrence network can visually show the co-occurrence relationship between two nodes that are directly connected, but second-order related words that are not directly connected are not easy to find, and interdisciplinary second-order related words are even more dazzling. In order to accurately and intuitively express the attributes of inter-disciplinary keyword co-occurrence nodes in the network, and at the same time facilitate the acquisition of potential interdisciplinary cooperation topics for each keyword through operation, this paper builds a twodimensional vector space model of network nodes and integrates The nodes are all expressed in the form of a vector matrix. Among them, the point adjacent to the node $\mathrm{Ni}$ is called the neighbor of the point; the keyword co-occurrence network is an undirected network, the size of the node in the network indicates the frequency of the keyword; the thickness of the edge indicates the weight of the edge between the two values, the weight represents the number of co-occurrences corresponding to two nodes.

As shown in Figure 1, ai represents the initial word node, bij is the neighbor of ai; ci is one or a few of the neighbors of bij, which corresponds in the recognition result. $\mathrm{Ljl}$ is the number of cooccurrences of the initial word ai and the middle word bij, and lj2 is the number of co-occurrences of bij and the target word $\mathrm{c}_{\mathrm{i}}$.

$$
a_{i}=\left[\begin{array}{cccc}
T_{i} & l_{11} & l_{12} & b_{i 1} \\
T_{i} & l_{21} & l_{22} & b_{i 2} \\
\cdots & \ldots & \ldots & \ldots \\
T_{i} & l_{n 1} & l_{n 2} & b_{i n}
\end{array}\right]
$$

In the two-dimensional vector space model, the first column Ti of the matrix is the frequency of the node keyword ai; the second column lj1 is the node and each neighbor; the third column bij is all neighbors of the node ai. In this representation, the relationship between each keyword ai and its neighbors bij is clear at a glance. At the same time, for open cross-disciplinary knowledge discovery methods to find potential interdisciplinary collaborative topics for each word, the neighbors in each keyword matrix are the candidate words corresponding to that word. Because some neighbor point ai relationship strength is small, so not every candidate word is suitable for intermediate term, it is necessary to point screening.

\subsection{Definition of Substitution Method to Identify Potential Interdisciplinary Collaborative Research Topics}

Since all of the words process is processed in the same uniform, the core vector in the keyword model obtained in the above procedure. In order to identify the corresponding A and C, based on the idea of an open unrelated knowledge discovery method, this paper proposes an operation method suitable for the keyword vector model-substitution method.Taking the second-order correlation matrix of the keyword ai and taking the knowledge graph as an example, the operation process of finding the knowledge graph by the substitution method is introduced in detail.

The substitution method is a process of substituting the core vector model of the middle word of each keyword into the core vector model of the word, and this process can be realized by Excel and VBA programming.Obviously, the above process of calculating the second order obtained related words only package includes interdisciplinary words, such as road network model, including the word in the same discipline, such as co-citation, there are two common words, such as big data.Therefore, in order to identify the interdisciplinary potential cooperation topics from all second-order keywords, it is necessary to further label the source of the subject after each keyword, and use the VBA program to uniformly filter all the second-order correlation matrices after the operation. After the screening, the middle word bij in the second column of the matrix is the cross- 
study keyword of the two disciplines, and the fourth column is a word that is different from the source discipline of the keyword ai, that is, the potential interdisciplinary cooperation subject term ci. The keyword knowledge graph of informatics, through the cross-disciplinary study of keyword data mining, establishes a link with the computer science keyword road network model. As shown in Figure 1, the road network model is a potential interdisciplinary research theme of the knowledge graph.

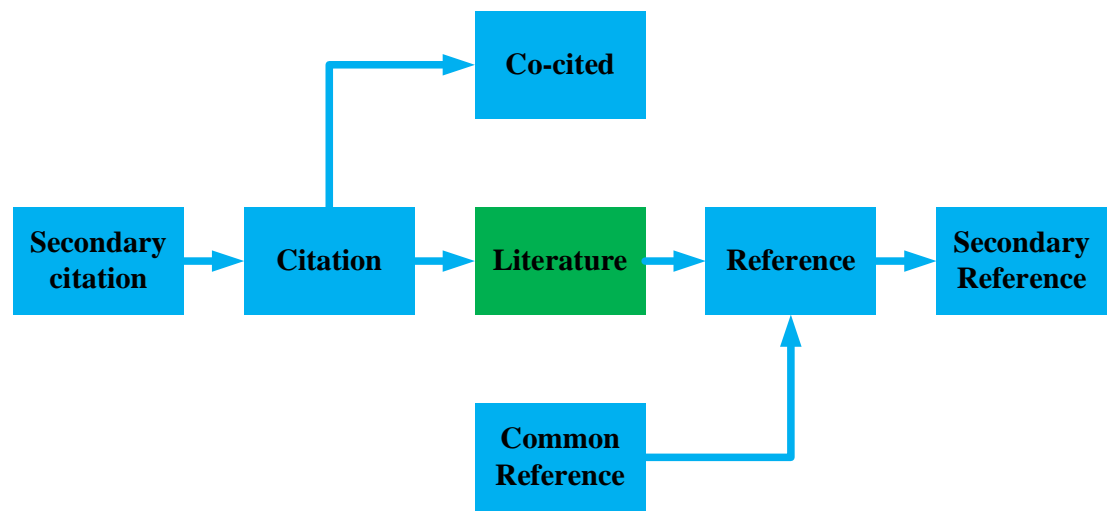

Figure1 Schematic Diagram of Citation Network

\subsection{Calculate the Cooperation Potential of Potential Interdisciplinary Cooperation Topics}

In the above process, all nodes ai in the interdisciplinary keyword co-occurrence network are expressed in matrix form by establishing a two-dimensional vector space model of the undirected network. After a certain rule, the intermediate of ai is selected and the core vector model of ai is established. The computer technology potential cooperation theme is identified through substitution method and further screening. Due to the different number of co-occurrences, the cooperation potential of the identified potential pairs will also be different.

Internet public opinion has great potential for interdisciplinary cooperation identified by closed non-relevant knowledge discovery methods, and the target words for cooperation identified in computer science are also the same. The research results of the two methods have the same part, which shows that the open and closed non-related knowledge discovery methods are completely different, but the two are basically the same. The recognition results of the two methods are different, which shows that the two have their own emphasis in the research process and have room for mutual reference.

\section{Application and Analysis}

\subsection{The Mechanism of Topic Association Discovery from the Perspective of Knowledge Flow}

It analyzes the application of several pairs of potential cooperation themes with great potential for interdisciplinary cooperation, and discusses the technical solutions to solve the problems of information science using computer theory methods. Among them, network public opinion and subtopics have been analyzed and will not be repeated here.

This discovery method finds that the road network model in the field of computer science can cooperate with the informatics. Scientific knowledge map drawing, mining, analysis and display of scientific and technological knowledge and the interrelationship between these scientific and technological knowledge, promote the cooperation and deepening of scientific and technological research.The road network model is a research direction in the field of intelligent transportation. Among them, the mobile global mode and the mobile process mode can not only effectively find hot spots, but also give the global movement information of the observation point and the speed of a specific activity section.Knowledge graphs and road network models both display information in the form of images, and both can provide scientific research data for scientific research work through data mining.In the process of knowledge graph data mining, such as citation analysis, interdisciplinary development history of disciplines, etc., the technology and methods of the road 
network model can be used to establish and analyze the corresponding network to reveal the cognitive and development process of knowledge.

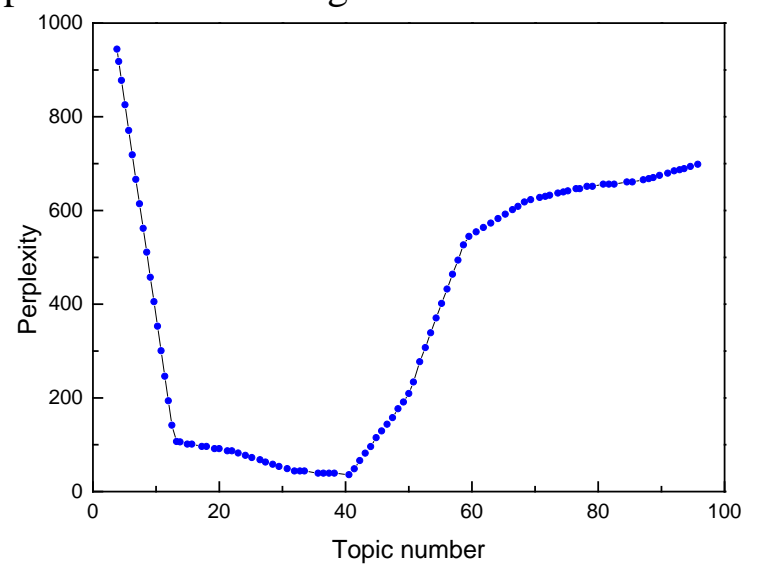

Figure 2 Topic-Confusion Map

In the recognition results, mouse behavior is a potential collaborative research topic of knowledge services in the computer field. Knowledge service has become the focus of attention in the field of library and information in recent years, and also the focus of library work.As shown in Figure 2, it proposed a retrieval satisfaction analysis and measurement technology. The basic principle of this technology is to use the mouse's sliding trajectory on the screen to calculate its virtual physical momentum. The coordinated relationship between them indicates that the user controls the biological energy generated during the mouse gliding and tending to click, and then maps this energy to a cognitively driven quantitative index of satisfaction, which assists the measurement of satisfaction. Knowledge services are inseparable from information retrieval, and the behavior of the mouse can measure the satisfaction of users during the information retrieval process. Therefore, applying the principle of mouse energy conversion to knowledge services can just solve the measurement of user satisfaction with library knowledge services problem.

Through open and unrelated knowledge discovery methods, the popularity of projects in the computer field can be interdisciplinary cooperation with information recommendation.Information recommendation refers to information that the user recommends that the user may be interested in users. Collaborative filtering has two main algorithms in information recommendation, which are based on items and users, that is, recommending to users by finding the correlation between items or users. The popularity of a project can reflect the degree of attention of a project. From the perspective of collaborative filtering, if the popularity of a certain project is very low, and it happens that the target user is highly relevant to the project, this can reflect the user's Project interest, the project popularity idea can more accurately analyze the user's interest. Therefore, the idea will help improve the accuracy of information recommendation.

\subsection{Knowledge Subject Discovery Mechanism for Cooperative Portfolio Relationship Prediction}

As the core of knowledge, the subject in the literature is a direct representation of knowledge. The citation relationship between the documents essentially reveals the flow and transfer of knowledge, so it has a wide range of applications in describing the knowledge flow network. Therefore, the topic-level 3D citation association network constructed in this paper can be regarded as a kind of knowledge flow network from the perspective of 3D citation association fusion. The process of flow and transfer between research topics in the field of knowledge flow network representation ${ }^{[6]}$. As a representation of multi-knowledge fusion innovation, it can excavate research frontiers or emerging trends in specific fields. In this study, the research method of link prediction is used to realize the discovery of future topic associations, that is, the realization of potential knowledge flow prediction ${ }^{[7]}$.

The so-called potential knowledge flow, also known as the future knowledge flow, refers to the knowledge flow that has not yet been generated but is likely to occur in the future, and can be used 
to reveal the future knowledge innovation in the academic field.The detection of potential knowledge flow helps researchers to track research frontiers and emerging research trends, and provides a certain degree of reference for researchers' future research directions ${ }^{[8]}$.The author is the subject of knowledge, that is, the creator of knowledge.Association discovery between knowledge subjects is the study of association discovery between author objects. The most extensive application of association discovery between authors is to study the author's cooperative relationship prediction, which can find partners for scientific researchers and recommend suitable co-authors.

Corresponding to the commonly used method of studying the similarity between authors, the coauthor strength between authors is used to express the similarity of each other's research content. Direct citation of authors based on citation theory, co-citation of authors, or co-citation analysis of author literature, etc. The strength of citation or co-citation indicates the similarity of the research content. Many different types of author association networks mentioned above can be used to study the similarity of authors.

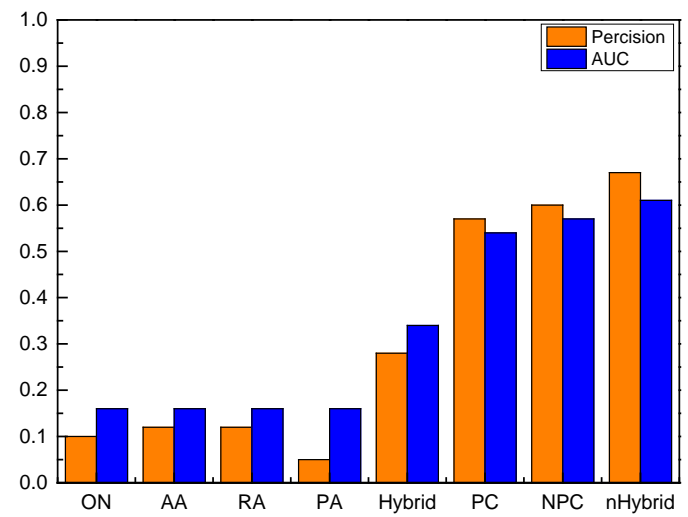

Figure 3 Recognition Results of Indicators under the Regression Model

As shown in Figure 3, the prediction of co-authoring relationships is mainly based on a single co-authoring network, with the authors as nodes, and the co-authoring relationship between authors as edges, using the theory of link prediction to extract some topological feature indicators to achieve future co-authoring relationships. However, there are few studies on the prediction of co-authoring relationships by comprehensive associations on the comprehensive use of three-dimensional citation associations to predict co-authoring relationships.

The knowledge flow network mapped by the three-dimensional citation association network can comprehensively reveal the current status of knowledge exchange and knowledge transfer in the field, but researchers and scientific management personnel are more interested in the future. When the knowledge in one topic is transferred to other topics, it will be internalized into new knowledge, and the transformation of innovative models will be carried out, and then become a research hotspot or research frontier in the field.The link prediction method is to analyze the topological characteristics of the social network to evaluate the possibility of generating links between two nodes in the network. It is the most widely used method to predict whether there will be links between nodes in the future.To some extent, the prediction of potential knowledge flow is the prediction of future links between topics.

We use link prediction metrics to measure the likelihood of links between pairs of directed topics. This paper uses link prediction methods to analyze the knowledge flow in the network, that is, the knowledge flow that may occur in the future, to explore the new knowledge fusion trends that may occur in the future, and to predict future research hotspots or research frontier issues. This paper by extraction from various types of path associated with the network to indicate more association between the author and the binding function.

\section{Conclusions}

Based on the perspective of three-dimensional citation association fusion, this paper explores the possible knowledge association discovery in the future, and aims to provide a reference for 
scientific research researchers in the field to choose partners and choose future research directions in order to obtain higher levels of innovation. At the same time, this article also made a certain degree of innovative attempts and explorations in the research ideas. As an attempt to discover knowledge correlation, this work can hope to cause scientific researchers to explore knowledge correlation under citation correlation and many other directions. Wide and deeper attention.

In this paper, based on the interdisciplinary keyword co-occurrence network, a two-dimensional vector space model representation method of nodes in an undirected network is constructed, and the substitution method operation of the two-dimensional vector space model is defined.Through the analysis of the identified cooperation topics, it is found that the recognition results of the open unrelated knowledge discovery method proposed in this paper do have certain interdisciplinary cooperation significance and are theoretically feasible.Although the identification of potential interdisciplinary collaborative research topics can discover interdisciplinary solutions to certain problems, it is only the first step in direction.For the development of interdisciplinary cooperation, it is also necessary to determine the appropriate interdisciplinary collaborators. Therefore, future research should be based on the identification of interdisciplinary collaborators, so as to make the identification of potential interdisciplinary cooperation topics more practical.

\section{References}

[1] Ding, Pingjian, et al. "Ensemble Prediction of Synergistic Drug Combinations Incorporating Biological, Chemical, Pharmacological, and Network Knowledge”. IEEE Journal of Biomedical and Health Informatics, vol. 23, no. 3, pp. 1336-1345, 2019.

[2] Liu, Xuejing, et al. "Knowledge-Guided Pairwise Reconstruction Network for Weakly Supervised Referring Expression Grounding." Proceedings of the 27th ACM International Conference on Multimedia, vol. 11, no. 2, pp. 539-547, 2017.

[3] Grillitsch, Markus, et al. "Unrelated Knowledge Combinations: The Unexplored Potential for Regional Industrial Path Development”. Cambridge Journal of Regions, Economy and Society, vol. 11, no. 2, pp. 257-274, 2018.

[4] Mayo, Joseph A. "Socially Constructed Knowledge: Using Cooperative Learning in Assessment Instruction”. Pedagogy and the Human Sciences, vol. 3, no. 1, pp. 52-64, 2013

[5] Catarci, Tiziana, and Maurizio Lenzerini. "Representing and Using Interschema Knowledge in Cooperative Information Systems". International Journal of Cooperative Information Systems, vol. 2, no. 4, pp. 375-398, 1993.

[6] Daoutis, Marios, et al. “Cooperative Knowledge Based Perceptual Anchoring”. International Journal on Artificial Intelligence Tools, vol. 21, no. 3, pp. 125-132, 2012.

[7] Lahra, Monica M., et al. "Cooperative Recognition of Internationally Disseminated CeftriaxoneResistant Neisseriagonorrhoeae Strain”. Emerging Infectious Diseases, vol. 24, no. 4, pp. 735-740, 2018.

[8] López-Rodríguez, Patricia, et al. "Non-Cooperative Target Recognition by Means of Singular Value Decomposition Applied to Radar High Resolution Range Profiles”. Sensors, vol. 15, no. 1, pp. 422-439, 2014. 